\title{
Comparison of effect of aquatic interventions on cardiac modulation of obese young males in motion. A crossover trial
}

\author{
Ertan Tufekcioglu ${ }^{1}$, Ferman Konukman ${ }^{2}$, Samer Arafat $^{3}$, Abdul-Majeed Almalty $^{4}$, \\ Abdussalam Kanniyan ${ }^{1}$, Bijen Filiz ${ }^{5}$
}

${ }^{1}$ Physical Education Department, King Fahd University of Petroleum \& Minerals, Dhahran, Saudi Arabia; ${ }^{2}$ Department of Physical Education, Qatar University, Doha, Qatar; ${ }^{3}$ Information and Computer Science Department, King Fahd University of Petroleum \& Minerals, Dhahran, Saudi Arabia; ${ }^{4}$ Physical and Occupational Therapy Department, The Hashemite University, Zarqa, Jordan; ${ }^{5}$ Faculty of Sport Sciences, Kocatepe University, Afyon, Turkey

\begin{abstract}
Study aim: The study aimed to compare the effects of passive Watsu therapy and immersion on cardiac locomotor synchronization of obese young males.

Material and methods: Twenty-six volunteer obese males participated in this study (age 18.3 \pm 0.32 , BMI 36.9 \pm 6.52 ). Heart rate variability parameters were recorded in different positions by the Polar $\mathrm{H} 7$ heart rate sensor and HRV+ software. Participants were assigned to two groups, randomly, in a single-blinded crossover design. Kubios HRV 2.2 and MATLAB were used to analyze the bio-signals. Statistical analysis was performed via t-test and ANOVA (analysis of variance) using SPSS. For the significance in results and group comparison, the paired t-test and the independent t-test were used respectively.

Results: Combined results indicated that Watsu therapy increased $3 \mathrm{HRV}$ vertical position parameters and immersion increased 3 HRV non-locomotor parameters, significantly $(\mathrm{p}<0.05)$.

Conclusion: The findings show that Watsu and immersion improved the specific autonomic cardiac modulation. However, non-contact immersion seemed to provide better synchronization of cardiac control and locomotion. The close contact Watsu approach provided improvements in autonomic cardiac regulation. Collectively, these improvements suggest the combination of both therapies in maximizing the cardiac benefits sought by aquatic therapy programs.
\end{abstract}

Keywords: Heart rate variability - Watsu - Immersion - Obesity - Locomotion - Synchronization

\section{Introduction}

The increase in the prevalence of obesity has reached pandemic levels in the last 50 years [3]. If current trends continue, it is estimated that 2.7 billion adults will be overweight, over 1 billion affected by obesity, and 177 million adults severely affected by obesity by 2025 [47].

Obesity plays a profound role in risk of death from COVID-19, particularly in male patients and younger populations [42]. For every increase from one body mass index (BMI) category to the next, there was a $70 \%$ increased risk of mortality [34]. Obesity prevalence for a population is quantified by body mass index (BMI). Higher BMI constitutes a significant risk for noncommunicable diseases such as cardiovascular disease, diabetes mellitus, and musculoskeletal disorders, which were the leading causes of death in 2012 [24].
Obesity is defined based on BMI values $(\geq 30)$ and affects the dynamic interactions between the autonomic nervous system (ANS), locomotor, and cardiovascular systems [17]. Obesity shows heterogeneity [29]. Distribution of body fat may be the factor causing inconsistency of the results for the BMI-HRV relation [45]. The excessive accumulation of fat tissues around the waist area leads to morphological changes in the cardiac structure leading to overt cardiovascular diseases [36]. The obesity-induced multifactorial mechanisms of increased ANS imbalance are not explicit [2]. However, higher parasympathetic heart control causes cardioprotective ANS modulation [20].

Aquatic therapies have improved a variety of conditions [4, 28, 39] including ANS modulation [7, 23]. In the history of aquatic therapy, Watsu is a novel body-based modality with growing acceptance in interdisciplinary treatment and aquatic therapy programs. Watsu comprises 
stretching, joint mobilization, vertebral traction, trunk rotation, deep breathing and acupressure while being floated in the arms of a practitioner [11]. The dyadic nature of Watsu therapy requiring coordination between active implementation (therapist) and passive receipt (client) of the technique is likely to promote specific cardiac and respiratory patterns considering the improvements in the previous studies $[5,25,43]$.

On the other hand, immersion has been used as a hydrotherapy or balneotherapy method in water with varying temperatures for centuries. Immersion provides thermal and mechanical therapeutic properties of water (buoyancy, viscosity, and hydrostatic pressure) that can enhance blood flow (vasodilation) and cardiovascular activity [33]. However, the effect of partial or complete immersion of the body needs to be further investigated [1].

The effect of aquatic interventions on autonomic activity, autonomic control during motor tasks and emotional correlations can be analyzed using heart rate variability (HRV) parameters [16]. HRV is noninvasive and amenable, with promising quantitative biomarkers of ANS activity, and is defined as the variation of beat-to-beat R-R intervals recorded by an electrocardiogram [38]. HRV is used in risk stratification of cardiac and diabetic patients and the follow-up of patients after therapeutic interventions [27]. An inverse relationship between BMI and HRV was found in young populations [46].

The majority of the research has studied post-exercise stationary HRV recordings based on the intensity and duration of exercises (12). Simply, there is a lack of studies to clarify even the effect of basic human locomotion on HRV. However, a study suggested that enhanced non-stationary HRV recordings are likely to increase our understanding of the dynamic adaptability of autonomic cardiac modulation in a variety of motor tasks and ANS capacity in responding to physiologic challenges [22]. Considering the cardio locomotor coupling due to pedaling or walking stride frequency, walking HRV, i.e. cardio locomotor synchronization (CLS), and cycling HRV, i.e. cardio non-locomotor synchronization (CnLS), can reveal the specific autonomic cardiac modulation in motion [19]. Moreover, motor performance can be estimated via CLS and CnLS parameters more accurately than stationary HRV recordings.
In this regard, the rhythm of muscle contractions entraining the cardiac cycle during locomotion is one of the physiologic afferent input patterns affecting ANS activity [32]. This entrainment between the heartbeat and locomotor rhythm can optimize blood flow to muscles and minimize the cardiac load enhancing CLS in walking [30]. Peak intra-arterial pressure due to the cardiac contraction likely occurs at the lowest phase of the intramuscular pressure cycle [41] as CnLS in cycling. The effectiveness of aquatic interventions on the complex mechanism of autonomic cardiac regulation in motion (CLS and CnLS) among obese participants needs to be investigated.

Although there are limited functional qualitative studies related to HRV, obesity, and aquatic therapies, the empirical evidence to eliminate the methodological problems inherent in the analysis of HRV is not sufficient $[15,38]$. The previous study results are difficult to compare due to the absence of motion-related HRV and the differences in study populations and the recording conditions.

We hypothesized that Watsu and immersion are beneficial for the ANS modulation of obese youth in motion. Thus, this study aimed to analyze and compare the specific effects of Watsu therapy and immersion protocols on autonomic cardiac modulation.

\section{Material and methods}

\section{Participants}

Participants were 26 volunteers, sedentary, obese, but otherwise healthy males recruited from first-year students at a college. They were not taking any medication and had no history of any known disease affecting cardiovascular health.

Participants were divided into Watsu first (WI, $\mathrm{n}=13$ ) and immersion first (IW, $\mathrm{n}=13$ ) groups, randomly, in a crossover design. After the baseline recordings, in the $1^{\text {st }}$ period, the WI group received Watsu therapy, whereas the IW group received immersion, twice a week, for five weeks. After a 3-week washout period with no intervention applied, the groups crossed over from receiving Watsu to immersion and vice versa for the $2^{\text {nd }}$ period. Participants were instructed against taking caffeine or exercising for the whole day before the recordings.

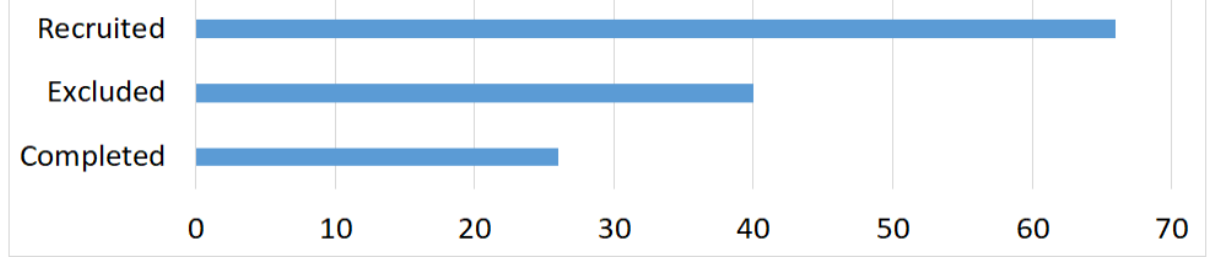

Figure 1. Number of participants in the phases of the study 
A medical history of cardiac disease, drugs affecting the cardiac modulation taken up to 15 days before the baseline recordings, infectious pathologies, or any disease known to affect the autonomic cardiac function were the exclusion criteria of the study.

Participants who fulfilled the initial inclusion criteria were subsequently excluded for the following reasons: late presence and first absence (\#28), observation of fatigue, sensitivity to pool chemicals (\#12) in the intervention period (Fig. 1).

All experimental procedures and methods were explained in detail to each subject, who then signed the informed consent. The ethic committee formed by the Deanship of Scientific Research at the university approved the study. The study was conducted following the World Medical Association Declaration of Helsinki, revised in 2000.

\section{Recording devices for body composition, blood pressure and HRV}

Body composition was analyzed using a direct segmental multi-frequency bioelectrical impedance (InBody370) analyzer (InBody Co., Ltd. Seoul, Korea). Blood pressure (BP) was measured using a digital BP apparatus (Omron Healthcare Co. Ltd., in Matsusaka Mie, Japan).

Stationary and non-stationary R-R intervals were measured by the Polar H7 Bluetooth Heart Rate Sensor \& Fitness Tracker (Polar Electro Oy, Kempele, Finland). The sensors connected to the strap received and transmitted signals to the iPad mini 4 (Apple Inc, CA, USA) which has digital standardized HRV signal processing software $(\mathrm{HRV}+)$ that fully supports Bluetooth (4.0) Smart connectivity. The validity [35] and reliability of Polar $\mathrm{H} 7$ are confirmed in rest and active conditions [12]. The participants were seated in a comfortable recliner chair for 10 minutes. Next, BP was measured, followed by HRV baseline measurements for 5 minutes in each position and locomotion, successively (supine-horizontal, sitting-vertical, walking-locomotor and cycling-non-locomotor). The 5-minute recording is the main data tool for the literature on HRV correlating with 24 $\mathrm{h}$ HRV recording [38]. The $5 \mathrm{~min}$ recording length is suggested for reliable HRV indices to evaluate physiological changes $[15,35]$. It is concluded that clinical and optimal performance progress induced by the interventions could be assessed using short-term HRV indices when clients breathe at regular rates $(\sim 11-20 \mathrm{bpm})$ [38].

Although the need for more investigation on CLS and CnLS in the literature is obvious, there is a methodological framework, which includes respiratory information, and identifies spectral components [18]. The validated CLS and CnLS analysis derived from R-R series (5 min recordings) in walking (at $4.5 \mathrm{~km} / \mathrm{h}$ ) and in cycling (at $50 \mathrm{~W}$ with a 55 -rpm pedal rate) were investigated in the current study using a Landice treadmill model L-7, and a Monark, model 686-cycle ergometer, respectively.
The corresponding data files were then transmitted to a personal computer as text files. Kubios HRV 2.2 and MATLAB (Version 2012b, MathWorks, Natick, USA) were used to analyze the HRV parameters, which were determined based on the decomposition of frequency bands (using either the fast Fourier transformation or the autocorrelative algorithms) of NN intervals. Kubios low power filter were performed to eliminate artifacts and ectopic beats in the recordings.

Time and frequency domain HRV indices used in the study are mean RR (the average of R-R intervals in $\mathrm{ms}$ ), RMSSD (root mean square successive difference between adjacent R-R in ms), pNN50 (\%) (the percentage of adjacent $\mathrm{NN}$ intervals differing by more than $50 \mathrm{~ms}$ in the recording), LF (the frequency band in the range $0.04-0.15 \mathrm{~Hz}$ ), HF (the frequency band in the range $0.15-0.4 \mathrm{~Hz}$ ), and VLF (the frequency band in the range 0.003-0.04 Hz).

Specifically produced water-soluble electrode gel for heart rate monitors was used on the skin of the participants to increase conductivity based on the instructions of the Polar H7 system chest strap. The chest strap and heart rate sensor were fitted around every participant's chest located in the middle area below the nipples. Participants were seated in a comfortable recliner chair for 10 minutes, after which BP was measured, followed by HRV baseline measurements. Standard spectral analysis was applied to the 5-minute HRV recording correlating with the 24-hour recording in horizontal (lying supine), vertical (sitting), locomotor (walking), and non-locomotor (cycling) positions. The average respiratory rate of the participants was 13.2 (SD 1.88) in steady recordings. Measurements were conducted between 5:00 and 7:00 pm in a quiet, low-light room with ambient temperature $\left(23 \pm 1^{\circ} \mathrm{C}\right)$ and humidity $(30 \pm 5 \%)$ one day after and before the experimental phases.

\section{Intervention protocol}

Two certified practitioners applied Watsu therapy to the WI group in the first and IW group in the second period. Specifically manufactured floating devices were tied over participants' thighs before Watsu sessions for better flotation. The techniques in Watsu were offerings, free spine, accordion, twist over, chest opening, corner spread and rotating accordion for the trunk rotation. The IW group in the first period and the WI group in the second period received shoulder-deep warm water immersion in the crossover design. The five-week periods consisted of two sessions (30 $\mathrm{min})$ per week. The temperatures of the pool water and air were $34^{\circ} \mathrm{C}$ and $30^{\circ} \mathrm{C}$, respectively.

\section{Statistical analysis}

Statistical analysis was performed via t-test and ANOVA (analysis of variance) using SPSS. The repeated ANOVA test was used for comparing and observing the 
unlikely period effect. The paired t-test and independent t-test were used to compare the difference within and between the groups. Cohen's d calculation was used to calculate the effect size. If the value of $d$ is smaller than 0.2 , the effect size is defined as very small, if it is between 0.3 and 0.5 , it is a medium effect, if it is between 0.6 and 0.8 , it is a large effect, and if it is greater than 0.8 , it is a very large effect [9]. The level of significance for all tests was set at $5 \%(\alpha=0.05)$.

\section{Results}

The physical characteristic differences of the groups were not significant. ( $\mathrm{p}=0.489$; height $\mathrm{t}(24)=0.574$, $\mathrm{p}=0.564$; weight $\mathrm{t}(24)=0.144, \mathrm{p}=0.449$; body mass in$\operatorname{dex} \mathrm{t}(24)=0.343, \mathrm{p}=0.244$; age $\mathrm{t}(24)=0.704, \mathrm{p}=0.882$; $\%$ body fat $\mathrm{t}(24)=0.589, \mathrm{p}=0.483$, basal metabolic rate $\mathrm{t}(24)=0.664, \mathrm{p}=0.474$, waist hip ratio $\mathrm{t}(24)=0.843$, $\mathrm{p}=0.523$ ) (Table 1). The difference between the group's baseline HRV values was not significant.

According to the combined results immersion increased non-locomotor RMSSD, pNN50 and mean R-R, significantly (Table 2) and no carryover effect was seen between the periods $(\mathrm{p}>0.05)$.

According to the combined HRV pre-posttest values of both periods for immersion, in non-locomotor synchronization (CnLS), the effect size value is very large according to RMSSD ( $\mathrm{d}=1.00)$, the effect size value is medium according to pNN50 $(\mathrm{d}=0.53)$, and the effect size is medium according to mean $\mathrm{RR}(\mathrm{d}=0.50)$ [9]. Watsu increased vertical HF, RMSSD, and pNN50, significantly $(\mathrm{p}<0.05)$ and horizontal, locomotor (CLS) and non-locomotor (CnLS) HRV values insignificantly (Table 3).

According to the period results, the WI group systolic $(136.8 \pm 12.3$ vs. $130.9 \pm 6.3)$ and diastolic $(80.7 \pm 9.6$ vs. $77.2 \pm 5.5$ ) blood pressure (BP) values decreased and the IW group systolic BP decreased $(135.1 \pm 10.2$ vs. $127.1 \pm 7.6 \mathrm{~mm} / \mathrm{Hg})$ significantly $(\mathrm{p}<0.01)$.

According to the combined HRV pre-posttest values of both periods for Watsu therapy, in vertical positions, the effect size value is medium according to RMSSD $(\mathrm{d}=0.39)$, the effect size value is medium according to pNN50 $(d=0.37)$, and the effect size value is medium according to HF $(d=0.53)$ [9]. The HRV parameters shown (Table 4) changed significantly $(\mathrm{p}<0.05)$.

According to HRV pre-posttest significant results of both periods, for the IW group in non-locomotor synchronization (CnLS), the effect size value is large according to HF $\left(n^{2}=0.60\right)$; for the IW group in vertical positions, the effect size value is very large according to VLF $\left(n^{2}=1.15\right)$, the effect size value is very large according to $\mathrm{LF}\left(\mathrm{n}^{2}=1.16\right)$, and the effect size value is large according to $\mathrm{HF}\left(\mathrm{n}^{2}=0.70\right)[9]$.

Table 1. Descriptive data of the groups (mean and standard deviation)

\begin{tabular}{lccccccc}
\hline Groups & Height & Weight & BMI & Age & \% Body fat & BMR & WHR \\
\hline IW $(\mathrm{n}=15)$ & $169.7 \pm 5.16$ & $107.1 \pm 21.2$ & $37.03 \pm 7.1$ & $18.3 \pm 0.34$ & $40.5 \pm 7.4$ & $1716.1 \pm 132.6$ & $1.001 \pm 0.07$ \\
WI $(\mathrm{n}=11)$ & $172.3 \pm 6.74$ & $109.2 \pm 21.8$ & $36.8 \pm 6.1$ & $18.2 \pm 0.29$ & $40.7 \pm 5.2$ & $1750.2 \pm 194.7$ & $1.033 \pm 0.09$ \\
\hline Total $(\mathrm{n}=26)$ & $171 \pm 5.95$ & $108.1 \pm 21.5$ & $36.9 \pm 6.6$ & $18.3 \pm 0.32$ & $40.6 \pm 6.3$ & $1733.2 \pm 163.7$ & $1.017 \pm 0.08$ \\
\hline
\end{tabular}

BMI: Body Mass Index; BMR: Basal Metabolic Rate. WHR: Waist-Hip Ratio (Mean \pm SD)

Table 2. Combined HRV pre-posttest values of both periods for immersion (*significant)

\begin{tabular}{lcccccccc}
\hline \multirow{2}{*}{$\begin{array}{l}\text { HRV } \\
\text { Indices }\end{array}$} & \multicolumn{2}{c}{ Horizontal } & \multicolumn{2}{c}{ Vertical } & \multicolumn{2}{c}{ Locomotor (CLS) } & \multicolumn{2}{c}{ Non-locomotor (CnLS) } \\
\cline { 2 - 9 } & Pre & Post & Pre & Post & Pre & Post & Pre & Post \\
\hline RMSSD & $0.05 \pm 0.03$ & $0.05 \pm 0.02$ & $0.04 \pm 0.03$ & $0.04 \pm 0.02$ & $0.02 \pm 0.01$ & $0.02 \pm 0.01$ & $0.02 \pm 0.01$ & $0.03 \pm 0.01 *$ \\
pNN50 & $26.12 \pm 21.15$ & $22.15 \pm 18.36$ & $18.71 \pm 18.47$ & $18.87 \pm 16.48$ & $3.50 \pm 7.96$ & $5.42 \pm 8.39$ & $2.43 \pm 4.23$ & $5.47 \pm 6.96 *$ \\
VLF & $48.84 \pm 17.74$ & $51.58 \pm 16.18$ & $60.04 \pm 20.23$ & $60.71 \pm 15.76$ & $63.30 \pm 18.51$ & $58.66 \pm 18.72$ & $61.81 \pm 18.55$ & $58.93 \pm 16.79$ \\
LF & $30.28 \pm 8.62$ & $31.33 \pm 11.75$ & $26.53 \pm 14.72$ & $28.44 \pm 15.18$ & $33.28 \pm 16.20$ & $29.60 \pm 15.98$ & $31.28 \pm 13.75$ & $33.56 \pm 14.40$ \\
HF & $20.67 \pm 15.76$ & $16.96 \pm 13.77$ & $13.37 \pm 11.15$ & $10.79 \pm 6.74$ & $7.99 \pm 8.25$ & $7.07 \pm 4.96$ & $6.85 \pm 6.35$ & $7.47 \pm 5.13$ \\
MRR & $0.81 \pm 0.11$ & $0.79 \pm 0.11$ & $0.75 \pm 0.10$ & $0.74 \pm 0.10$ & $0.61 \pm 0.07$ & $0.62 \pm 0.09$ & $0.59 \pm 0.07$ & $0.63 \pm 0.09 *$ \\
\hline
\end{tabular}

* Significant $\mathrm{p}<0.05$. 
Table 3. Combined HRV pre-posttest values of both periods for Watsu therapy

\begin{tabular}{lcccccccc}
\hline \multirow{2}{*}{$\begin{array}{l}\text { HRV } \\
\text { Indices }\end{array}$} & \multicolumn{2}{c}{ Horizontal } & \multicolumn{2}{c}{ Vertical } & \multicolumn{2}{c}{ Locomotor (CLS) } & \multicolumn{2}{c}{ Non-locomotor (CnLS) } \\
\cline { 2 - 9 } & Pre & Post & Pre & Post & Pre & Post & Pre & Post \\
\hline RMSSD & $0.04 \pm 0.02$ & $0.05 \pm 0.04$ & $0.03 \pm 0.02$ & $0.04 \pm 0.03^{*}$ & $0.02 \pm 0.01$ & $0.02 \pm 0.02$ & $0.02 \pm 0.01$ & $0.02 \pm 0.02$ \\
pNN50 & $19.33 \pm 17.89$ & $21.60 \pm 23.36$ & $13.03 \pm 11.60$ & $19.04 \pm 19.22 *$ & $1.75 \pm 2.48$ & $6.20 \pm 12.66$ & $3.23 \pm 6.17$ & $6.35 \pm 10.57$ \\
VLF & $60.55 \pm 16.96$ & $55.27 \pm 18.15$ & $60.25 \pm 13.73$ & $53.35 \pm 16.06$ & $62.14 \pm 18.32$ & $62.74 \pm 13.99$ & $62.23 \pm 13.06$ & $64.33 \pm 18.23$ \\
LF & $25.88 \pm 10.55$ & $29.41 \pm 11.98$ & $30.83 \pm 11.33$ & $33.34 \pm 12.87$ & $31.09 \pm 11.77$ & $30.41 \pm 14.57$ & $31.34 \pm 12.35$ & $29.45 \pm 15.63$ \\
HF & $13.52 \pm 10.65$ & $15.23 \pm 13.33$ & $8.88 \pm 5.10$ & $13.26 \pm 10.63^{*}$ & $6.14 \pm 3.65$ & $7.42 \pm 6.07$ & $6.39 \pm 3.94$ & $6.19 \pm 4.45$ \\
MRR & $0.76 \pm 0.13$ & $0.79 \pm 0.13$ & $0.72 \pm 0.13$ & $0.76 \pm 0.14$ & $0.60 \pm 0.07$ & $0.62 \pm 0.08$ & $0.61 \pm 0.09$ & $0.62 \pm 0.08$ \\
\hline
\end{tabular}

* Significant $\mathrm{p}<0.05$.

\section{Discussion}

Our current study confirmed the hypothesis that immersion and aquatic bodywork, Watsu, can improve autonomic cardiac modulation in obesity. Moreover, in group results, immersion and Watsu showed significant benefits in some CnLS parameters. However, in combined results, Watsu showed its efficiency only for vertical HRV parameters. Considering improved BP, the use of both aquatic interventions for cardiovascular health is suggested in line with other studies $[21,45]$.

The motor function and cardiac (HRV) benefits of aquatic interventions [31, 32] have gained a new dimension (CnLS) with our study illuminating the potential entrainment of oscillatory neural systems, especially autonomic cardiac regulation in motion [37]. Thus, immersion seems to be a preparatory environment enhancing the ANS control in specific motion. Immersion might have increased the muscle blood flow more efficiently than Watsu due to higher hydrostatic pressure and less vasoconstriction $[7,23]$.

This increased blood flow might have played a critical role in the non-locomotor (cycling) task requiring longer muscle contraction time than the locomotor (walking) task. Thus, the match between muscle contraction and increased blood flow can improve CnLS [32]. This cardiac coupling mechanism is likely to further support the synchronization of the network of neural oscillators. Also, participants immersed up to their necks received plenty of vestibular inputs activating vestibular sensoryrelated systems due to water resistance and buoyancy. Thus, relative instability during immersion stimulates the body to balance actively by the muscular reflexes, which can result in improved non-locomotor functioning. The immersion-induced muscular reflexes, similar to voluntarily synchronizing gait with the cardiac cycle, can be beneficial for running performance due to the reduced heart rate and increased HRV [10]. Another contribution to the improved CnLS after immersion might be the higher serotonin levels helping rebalance ANS activity [6]. However, these CLS and CnLS characteristics need to be further studied.

In Watsu, being floated, stretched, rotated, and glided from one therapeutic body position to another has altered proprioceptive and tactile sensory inputs, contributing to better cardiac modulation $[28,43]$ and probably the main contributor to improved vertical HRV.

In the IW group the significant increase in vertical and non-locomotor HF and decrease in VLF after Watsu also indicate improved ANS activity. The current study with 5 weeks of aquatic interventions can further clarify the results in a similar study of the 12-week Watsu therapy in the lower water temperature of $32^{\circ} \mathrm{C}$ [44]. The current study provides a critical element, ideal water temperature $\left(34^{\circ} \mathrm{C}\right.$ thermo-neutral water) in the therapy application. Thus, high quality Watsu applications might have played a major role in the current study results within shorter experimental periods ( 5 weeks). The underlying reason for the results could be a better quality sensory input in ideal water temperature. However, increased recording frequency in future studies can confirm the notion that water temperature can play a critical role in the cardiac autonomic regulation in Watsu. In comparison to a previous study [44], implications such as aquatic intervention modification in less than 12 weeks may result in better autonomic cardiovascular modulation.

Thus, the stimulation by the Watsu practitioner and warm water (tactile and proprioceptive) can alter the rhythm of bodily systems, providing new afferent input to the medulla and cortex $[13,14,16]$. These inputs enhance not only ANS activity but also cerebellar-motor cortex collaboration and are vital in motor learning, i.e. psychomotor performance [40]. It is worth mentioning that another form of synchronization that can occur in Watsu-like movement-based group practices is cardiac arousal between active and passive participants [8]. This 


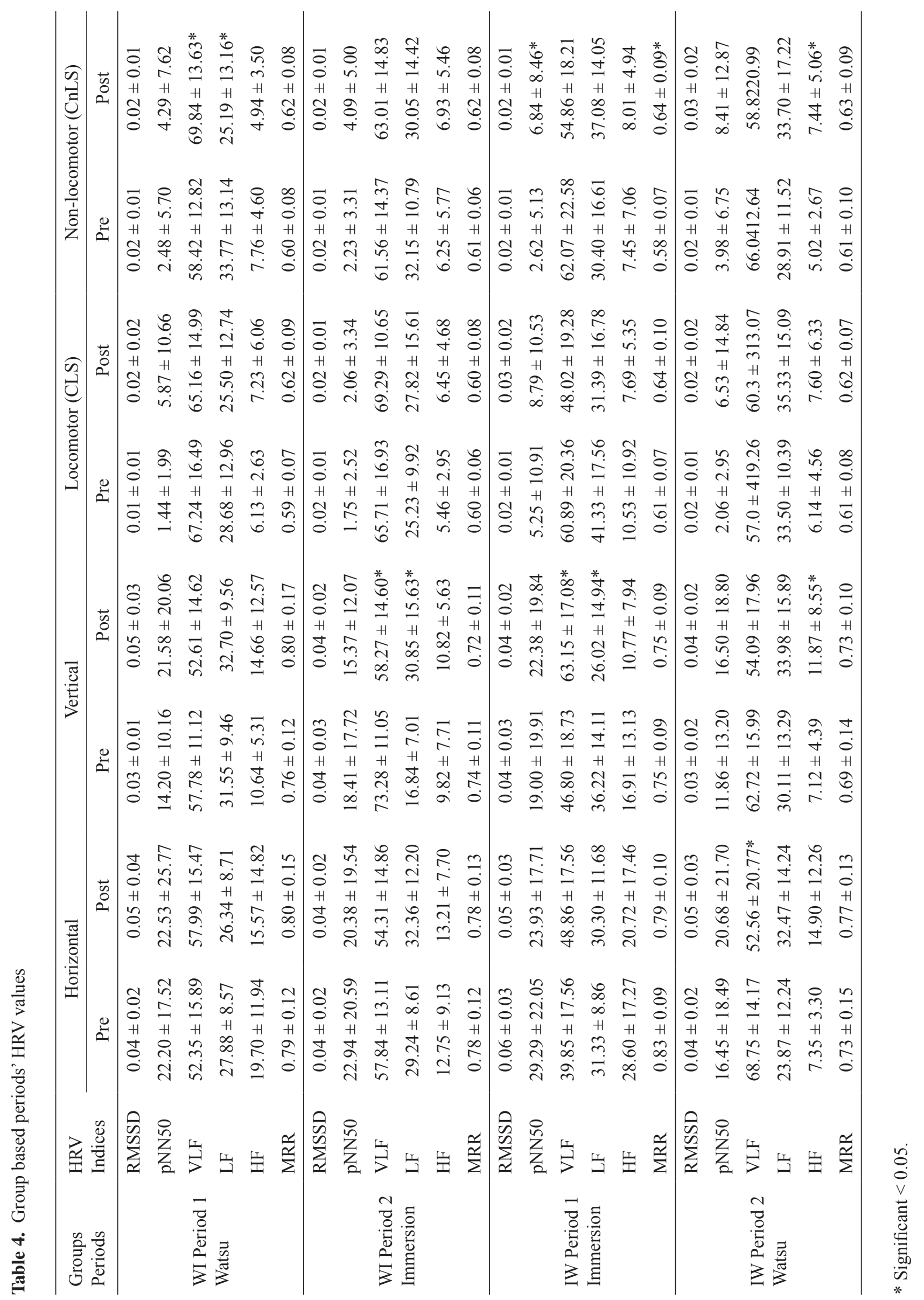


synchronization is most likely to happen during the passive interaction and participation in Watsu, resulting in increased flexibility of autonomic cardiac activity. The results (improved vertical HRV) might be the manifestation of this phenomenon. Also, manipulations on the shoulder and the neck can stimulate pressure receptors and the vagal activity, contributing to a higher physiological response capacity of ANS [26].

One of the potential limitations of the study was that the participants were mostly sedentary obese males. Other important limitations of the current study include not having the evaluation of multiple physiological recordings and emotional parameters. The dependence on the pre- and post-test results can be enriched with more frequent in-experiment and follow-up measurements. Third, the duration and triggering factors of obesity and activity level were not considered.

However, the significant HRV and blood pressure improvements indicate that the aquatic therapies may help reverse the effect of obesity-related issues such as cardiovascular dysregulation and hypertension. Use of both immersion and the interactive Watsu, together with the versatility of techniques used with no known side effects, is suggested. The R-R series recorded in stationary and non-stationary conditions could provide better diagnostic information for the autonomic cardiac regulation and can lead to further extrapolations. Thus, the current study results can form the basis for future post-aquatic therapy cardiac regulation studies for attentional interpretations and consistent implications.

Future large-scale randomized trials with standardized protocols and synchronized multiple physiological recordings (stationary and non-stationary) may help confirm the therapy-specific cardiovascular changes in obesity.

In conclusion, Watsu and immersion improved autonomic cardiac regulation and cardiac locomotor synchronization in obese youth. The revealed findings of the study suggest the incorporation of both aquatic therapies in wellness programs to improve autonomic cardiac regulation.

\section{Conflict of interest: Authors state no conflict of interest.}

\section{References}

1. An J., Lee I., Yi, Y. (2019) The Thermal Effects of Water Immersion on Health Outcomes: An Integrative Review. Int. J. Environmental Res. Pub. Health, 16(7): 1280.

2. Balasubramanian P., Hall D., Subramanian M. (2019) Sympathetic nervous system as a target for aging and obesity-related cardiovascular diseases. GeroScience, 41: 13-24.

3. Blüher M. (2019) Obesity: Global Epidemiology and Pathogenesis. Nat. Rev. Endocrinol., 15: 288-298.
4. Carayannopoulos A.G., Han A., Burdenko I.N. (2020) The benefits of combining water and land-based therapy. J. Exerc. Rehab., 16(1): 20-26.

5. Chen O., Grabarnick A., Pilz-Burstein R. (2018) The effect of Watsu treatments on pain indices and on the quality of sleep in women diagnosed as fibromyalgia patients. JIPTS, 20(2): 14-24.

6. Choi K.H., Kim J., Kwon O.S., Kim M.J., Ryu Y.H., Park J.E. (2017) Is heart rate variability (HRV) an adequate tool for evaluating human emotions? - A focus on the use of the International Affective Picture System (IAPS) Psychiatry Res., 251: 192-196.

7. Chouchou F., Pichot V., Costes F., Guillot M., Barthélémy J.C., Bertoletti L., Roche F. (2020) Autonomic cardiovascular adaptations to acute head-out water immersion, head-down tilt and supine position. Eur. J. Appl. Physiol., 120: 337-347.

8. Codrons E., Bernardi N.F., Vandoni M., Bernardi L. (2014) Spontaneous Group Synchronization of Movements and Respiratory Rhythms. PLoS ONE 9(9): e107538.

9. Cohen J. (1988) Statistical power analysis for the behavioral sciences, 2nd edition. Hillsdale, NJ: Erlbaum.

10. Constantini K., Stickford A., Bleich J.L., Mannheimer P.D., Levine B.D., Chapman, R. F. (2018) Synchronizing Gait with Cardiac Cycle Phase Alters Heart Rate Response during Running. Med. Sci. Sport Exerc., 50(5): 1046-1053.

11. Dull H. (2008) Watsu®: Freeing the Body in Water, Trafford Publishing, Victoria, Canada.

12. Esco M.R., Flatt A.A., Williford H.N. (2017) Postexercise heart rate variability following treadmill and cycle exercise: a comparison study. Clin. Physiol. Funct. Imaging, 37: 322-327.

13. Elbers J., McCraty R. (2020) HeartMath approach to selfregulation and psychosocial well-being. J. Psychol. Afr., 30(1): 69-79.

14. Gatti E., Calzolari E., Maggioni E., Obrist M. (2018) Emotional ratings and skin conductance response to visual, auditory and haptic stimuli. Sci. Data 5: 180120.

15. Georgiou K., Larentzakis A.V., Khamis N.N., Alsuhaibani G.I., Alaska Y.A., Giallafos E.J. (2018) Can Wearable Devices Accurately Measure Heart Rate Variability? A Systematic Review, Folia Med., 60(1): 7-20.

16. Godfrey K.M., Juarascio A., Manasse S., Minassian A., Risbrough V., Afari N. (2019) Heart rate variability and emotion regulation among individuals with obesity and loss of control eating. Physiol. Behav., 199: 73-78.

17. Hall J.E., do Carmo J.M., da Silva A.A., Wang Z., Hall M.E. (2015) Obesity-induced hypertension: interaction of neurohumoral and renal mechanisms. Circ. Res., 116(6): 991-1006.

18. Hernando D., Garatachea N., Almeida R., Casajús J.A., Bailón R. (2018) Validation of heart rate monitor polar 
rs800 for heart rate variability analysis during exercise. $J$. Strength Cond. Res., 32: 716-725.

19. Hernando D., Hernando A., Casajús J.A., Casajús J.A, Laguna P., Garatachea N., Bailón R. (2018) Methodological framework for heart rate variability analysis during exercise: application to running and cycling stress testing. Med. Biol. Eng. Comput., 56: 781-794.

20. Herring N., Kalla M., Paterson D.J. (2019) The autonomic nervous system and cardiac arrhythmias: current concepts and emerging therapies. Nat. Rev. Cardiol., 16: 707-726.

21. Hiromi M., Isao S., Eri E., Koutatsu M., Tadahiro K., Takeshi T. (2014) Heart rate variability and blood pressure among Japanese men and women: a communitybased cross-sectional study. Hypertens. Res., 37(8): 779-884.

22. Hsieh T.C., Huang C.J., Hung T.M. (2010) Relationships between heart rate variability, attention, and athletic performance, Int. J. Sport Exerc. Psychol., 8(4): 473-475.

23. Jiyeon A.,, Insook L., Yunjeong Y. (2019) The Thermal Effects of Water Immersion on Health Outcomes: An Integrative Review. Int. J. Environ. Res. Public Health. 16: 1280.

24. Lambert E.A., Esler M.D., Schlaich M.P., Dixon J., Eikelis N., Lambert G.W. (2019) Obesity-Associated Organ Damage and Sympathetic Nervous Activity A Target for Treatment? Hypertension, . 73: 1150-1159.

25. Maczkowiak S., Hölter G., Otten H. (2007) Watsu ${ }^{\circledR}$ - the effect of differently accentuated movement therapy interventions on clinically depressive patients BG, Corpus ID: 79223084. 23(2): 58-64.

26. Meier M., Unternaehrer E., Dimitroff S.J., Benz A., Bentele U.U., Wenzel M., Schorpp S.M., Pruessner J. (2020) In search of a standardized protocol for parasympathetic nervous system activation.

27. Metelka R. (2014) Heart rate variability - current diagnosis of the cardiac autonomic neuropathy. A review. Biomed. Pap. Med. Fac., 158(3): 327-338.

28. Mooventhan A., Nivethitha L. (2014) Scientific EvidenceBased Effects of Hydrotherapy on Various Systems of the Body. N. Am. J. Med .Sci., 6(5): 199-209.

29. Neeland I.J., Poirier P., Després J.P. (2018) Cardiovascular and Metabolic Heterogeneity of Obesity Clinical Challenges and Implications for Management Circulation. Circulation, 137: 1391-1406.

30. Niizeki K., Saitoh T. (2014) Cardio-locomotor phase synchronization during rhythmic exercise. J. Phys. Fit. Sports Med., 3(1); 11-20.

31. Noa R.B., Michal K., (2014) The Association between Cardiac Autonomic Control System and Motor Performance among Patients Post Stroke: Review of the Literature. Int. J. Neurorehabilitation, 1: 136.

32. Nomura K., Takei Y., Yanagida Y., (2003) Comparison of cardio-locomotor synchronization during running and cycling. Eur. J. Appl. Physiol., 89: 221-229.
33. Parker R., Higgins Z., Mlombile Z., Mohr M.J., Wagner T.L. (2018) The effects of warm water immersion on blood pressure, heart rate and heart rate variability in people with chronic fatigue syndrome. S. Afr. J. Physiother, 74(1): 442.

34. Pettit N.N., MacKenzie E.L., Ridgway J.P., Pursell K., Ash D., Patel B., Pho M.T. (2020) Obesity is Associated with Increased Risk for Mortality Among Hospitalized Patients with COVID-19. Obesity, 28: 1806-1810.

35. Plews D.J., Scott B., Altini M., Wood M., Kilding A.E., Laursen P.B. (2017) Comparison of Heart-Rate-Variability Recording With Smartphone Photoplethysmography, Polar H7 Chest Strap, and Electrocardiography. Int. J. Sports Physiol. Perform., 12(10): 1324-1328.

36. Poirier P., Martin J., Marceau P., Biron S., Marceau S. (2004) Impact of bariatric surgery on cardiac structure, function and clinical manifestations in morbid obesity. Expert Rev. Cardiovasc. Ther., 2(2): 193-201.

37. Rouffet D., Taylor S., Sparrow W., Begg R., (2008) Cardio-locomotor entrainment during walking in young healthy people. A preliminary study, International Conference on Intelligent Sensors, Sensor Networks and Information Processing 347-350.

38. Shaffer F., Ginsberg J.P. (2017) An overview of heart rate variability metrics and norms. Front. Public Health, 5: 258.

39. Schitter A.M., Fleckenstein J., Frei P., Taeymans J., Kurpiers N., Radlinger L. (2020) Applications, indications, and effects of passive hydrotherapy WATSU (WaterShiatsu) - A systematic review and meta-analysis. PLoS ONE 15(3): e0229705.

40. Spampinato D.A., Celnik P.A., Rothwell J.C. (2020) Cerebellar-Motor Cortex Connectivity: One or Two Different Networks? J. Neurosci., 40(21): 4230-4239.

41. Takeuchi S., Nishida Y., Mizushima T. (2015) Evidence of an association between cardiac-locomotor synchronization and lower leg muscle blood perfusion during walking. J. Phys. Ther. Sci., 27(6): 1819-1822.

42. Tartof S.Y., Qian L., Hong V., Wei R., Nadjafi R.F., Fischer H., Li Z., Shaw S.F., Caparosa S.L., Nau C.L., Saxena T., Rieg G.K., Ackerson B.K., Sharp A.L., Skarbinski J., Naik T.K., Murali S.B. (2020) Obesity and Mortality Among Patients Diagnosed With COVID-19: Results From an Integrated Health Care Organization. Ann. Intern. Med., 0003-4819, M20-3742. Advance online publication.

43. Tufekcioglu E., Cotuk H.B. (2009) Comparison of heart rate variability in different body position on land and in water. Nigde University J. Phys. Educ. Sports Sci., 3(3): 152-159.

44. Tufekcioglu E., Erzeybek M.S., Kaya F., Ozan G. (2018) The Effect of 12-Week Passive Aquatic Bodywork on Sympathovagal Balance of Obese Youth. J. Educ. Train. Stud., 6(2): 166. 
45. Virtanen R., Jula A., Kuusela T., Helenius H., VoipioPulkki. (2003) Reduced heart rate variability in hypertension: associations with lifestyle factors and plasma renin activity. J. Hum. Hypertens., 17: 171-179.

46. Windham B.G., Fumagalli S., Ble A., Sollers J.J., Thayer J.F., Najjar S.S., Griswold M.E., Ferrucci L. (2012) The Relationship between Heart Rate Variability and Adiposity Differs for Central and Overall Adiposity. $J$. Obes., 2012: 149516. DOI: 10.1155/2012/149516.

47. www.worldobesity.org/about/about-obesity/prevalenceof-obesity

\section{Received 08.08.2020}

Accepted 24.11.2020

(C) University of Physical Education, Warsaw, Poland

Acknowledgments

I gratefully acknowledge the funded support provided by the Deanship of Scientific Research at King Fahd University of Petroleum and Minerals through Internal Project number 141044 for this research. 